\title{
Supernova Nucleosynthesis in the early universe
}

\section{Nozomu Tominaga ${ }^{1}$, Hideyuki Umeda ${ }^{2}$, Keiichi Maeda ${ }^{3}$, Ken'ichi Nomoto ${ }^{3,2}$ and Nobuyuki Iwamoto ${ }^{4}$}

\author{
${ }^{1}$ Optical and Infrared Astronomy Division, National Astronomical Observatory, Mitaka, \\ Tokyo, Japan \\ email: nozomu.tominaga@nao.ac.jp \\ ${ }^{2}$ Department of Astronomy, School of Science, University of Tokyo, Bunkyo, Tokyo, Japan \\ ${ }^{3}$ Institute for the Physics and Mathematics of the Universe, University of Tokyo, Kashiwa, \\ Chiba, Japan \\ ${ }^{4}$ Nuclear Data Center, Nuclear Science and Engineering Directorate, Japan Atomic Energy \\ Agency, Tokai, Ibaraki, Japan
}

\begin{abstract}
The first metal enrichment in the universe was made by supernova (SN) explosions of population (Pop) III stars. The history of chemical evolution is recorded in abundance patterns of extremely metal-poor (EMP) stars. We investigate the properties of nucleosynthesis in Pop III SNe by comparing their yields with the abundance patterns of the EMP stars. We focus on (1) jet-induced SNe with various properties of the jets, especially energy deposition rates $\left[\dot{E}_{\text {dep }}=(0.3-1500) \times 10^{51} \mathrm{ergs} \mathrm{s}^{-1}\right]$, and (2) SNe of stars with various main-sequence masses $\left(M_{\mathrm{ms}}=13-50 M_{\odot}\right)$ and explosion energies $\left[E=(1-40) \times 10^{51} \mathrm{ergs}\right]$. The varieties of Pop III $\mathrm{SNe}$ can explain the observations of the EMP stars: (1) higher $[\mathrm{C} / \mathrm{Fe}]$ for lower $[\mathrm{Fe} / \mathrm{H}]$ and $(2)$ trends of abundance ratios $[\mathrm{X} / \mathrm{Fe}]$ against $[\mathrm{Fe} / \mathrm{H}]$.
\end{abstract}

Keywords. Galaxy: halo, gamma rays: bursts, nuclear reactions, nucleosynthesis, abundances, stars: abundances, stars: Population II, supernovae: general

\section{Introduction}

Long-duration $\gamma$-ray bursts (GRBs) have been found to be accompanied by luminous and energetic Type Ic supernovae [SNe Ic, called hypernovae (HNe)] (e.g. Galama et al. 1998). Although the explosion mechanism is still under debate, photometric observations (a "jet break", e.g. Frail et al. 2001) and spectroscopic observations (a nebular spectrum, e.g. Maeda et al. 2002) indicate that they are aspherical explosions with jet(s).

The aspherical explosions are indirectly suggested from the abundance patterns of extremely metal-poor $(\mathrm{EMP})$ stars with $[\mathrm{Fe} / \mathrm{H}]<-3 \dagger$ which are suggested to show nucleosynthesis yields of a single core-collapse SN (e.g. Beers \& Christlieb 2005). C-enhanced EMP (CEMP) stars have been well explained by faint SNe with large fallback (Umeda \& Nomoto 2005; Iwamoto et al. 2005; Nomoto et al. 2006; Tominaga et al. 2007b). Some CEMP stars show enhancement of Co and Zn (e.g. Depagne et al. 2002) that requires explosive nucleosynthesis under high entropy. In a spherical model, a high entropy explosion corresponds to a high energy explosion that inevitably synthesizes a large amount of ${ }^{56} \mathrm{Ni}$, i.e., a bright SN (e.g. Woosley \& Weaver 1995). This incompatibility will be solved if a faint $\mathrm{SN}$ is associated with a narrow jet within which a high entropy region is confined (Umeda \& Nomoto 2005).

$\dagger$ Here $[\mathrm{A} / \mathrm{B}] \equiv \log _{10}\left(N_{\mathrm{A}} / N_{\mathrm{B}}\right)-\log _{10}\left(N_{\mathrm{A}} / N_{\mathrm{B}}\right) \odot$, where the subscript $\odot$ refers to the solar value and $N_{\mathrm{A}}$ and $N_{\mathrm{B}}$ are the abundances of elements $\mathrm{A}$ and $\mathrm{B}$, respectively. 


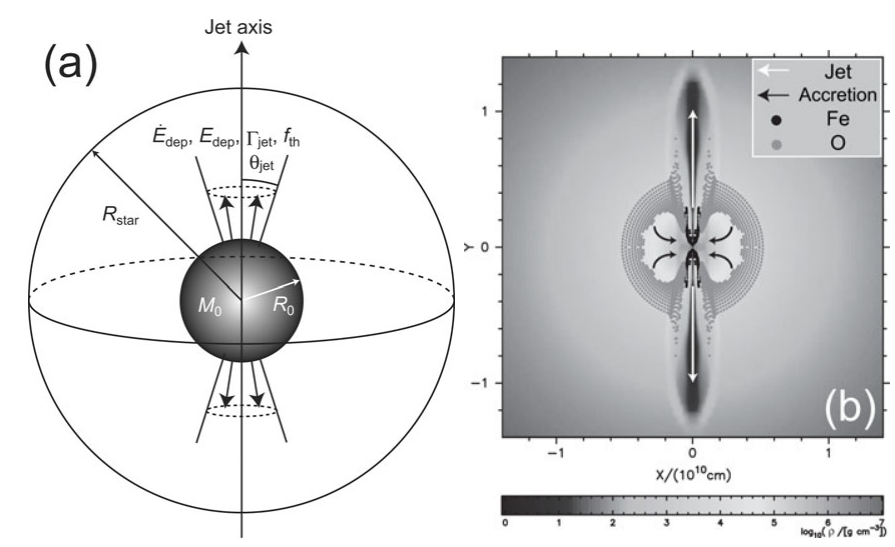

Figure 1. (a) Schematic picture of the jet-induced explosion. (b) Density structure of the $40 M_{\odot}$ Pop III star explosion model of $\dot{E}_{\mathrm{dep}, 51}=15$ at $1 \mathrm{sec}$ after the start of the jet injection.

\section{Models}

We investigate the jet-induced explosions (e.g. Maeda \& Nomoto 2003; Nagataki et al. 2006) of $40 M_{\odot}$ Pop III stars (Umeda \& Nomoto 2005; Tominaga et al. 2007b) using a two-dimensional special relativistic Eulerian hydrodynamic code (Tominaga 2007).

We inject the jets at a radius $R_{0}$, corresponding to an enclosed mass of $M_{0}$, and follow the jet propagation (Figs. 1ab). Since the explosion mechanism is unknown, the jets are treated parametrically with the following five parameters: energy deposition rate $\left(\dot{E}_{\mathrm{dep}}\right)$, total deposited energy $\left(E_{\text {dep }}\right)$, initial half angle of the jets $\left(\theta_{\text {jet }}\right)$, initial Lorentz factor $\left(\Gamma_{\text {jet }}\right)$, and the ratio of thermal to total deposited energies $\left(f_{\mathrm{th}}\right)$.

We investigate the dependence of nucleosynthesis outcome on $\dot{E}_{\text {dep }}$ for a range of $\dot{E}_{\mathrm{dep}, 51} \equiv \dot{E}_{\mathrm{dep}} / 10^{51} \mathrm{ergs} \mathrm{s}^{-1}=0.3-1500$. The diversity of $\dot{E}_{\mathrm{dep}}$ is consistent with the wide range of the observed isotropic equivalent $\gamma$-ray energies and timescales of GRBs (e.g. Amati et al. 2007). Variations of activities of the central engines, possibly corresponding to different rotational velocities or magnetic fields, may well produce the variation of $\dot{E}_{\mathrm{dep}}$. We expediently fix the other parameters as $E_{\mathrm{dep}}=1.5 \times 10^{52} \mathrm{ergs}$, $\theta_{\text {jet }}=15^{\circ}, \Gamma_{\text {jet }}=100, f_{\text {th }}=10^{-3}$, and $M_{0}=1.4 M_{\odot}\left(R_{0} \sim 900 \mathrm{~km}\right)$ in the models.

The hydrodynamical calculations are followed until the homologously expanding structure is reached $(v \propto r)$. The nucleosynthesis calculations are performed as post-processing with thermodynamic histories traced with marker particles that represent individual Lagrangian elements. In computing the jet composition, we assume that the jet initially has the composition of the accreted stellar materials.

\section{Jet-induced Supernovae}

\subsection{Fallback}

Figure 2a shows "accreted" regions for models with $\dot{E}_{\mathrm{dep}, 51}=120$ and $1.5 \mathrm{ergs} \mathrm{s}^{-1}$, where the accreted mass elements are initially located in the progenitor. The inner matter is ejected along the jet-axis but not along the equatorial plane. On the other hand, the outer matter is ejected even along the equatorial plane, since the lateral expansion of the shock terminates the infall as the shock reaches the equatorial plane.

The remnant mass $\left(M_{\text {rem }}\right)$ is larger for lower $\dot{E}_{\text {dep }}$. This stems from the balance between the ram pressures of the injecting jet $\left(P_{\text {jet }}\right)$ and the infalling matter $\left(P_{\text {fall }}\right)$. In order to inject the jet, $P_{\text {jet }}$ should overcome $P_{\text {fall }} . P_{\text {jet }}$ is determined by $R_{0}, \dot{E}_{\text {dep }}, \theta_{\text {jet }}, \Gamma_{\text {jet }}$, and 

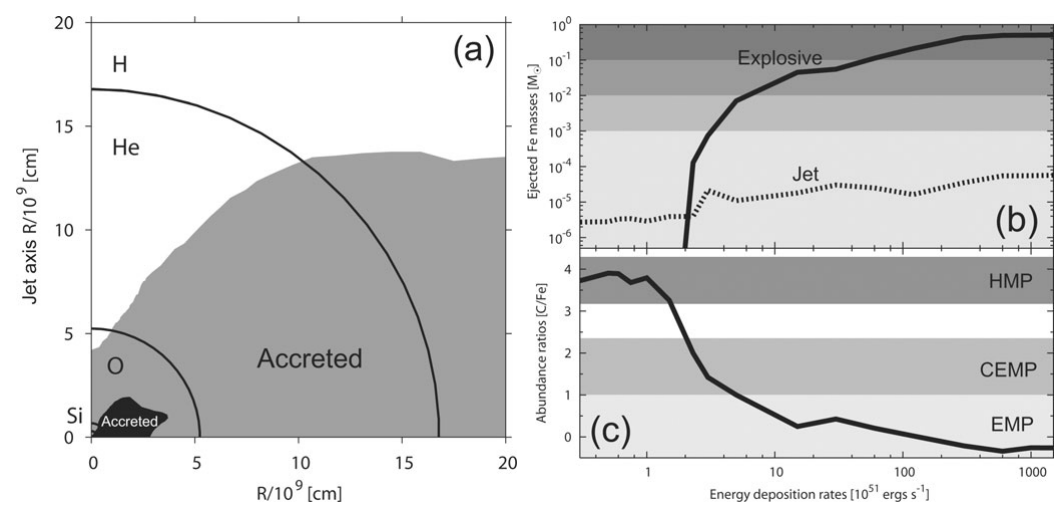

Figure 2. (a) Initial locations of the mass elements which are finally accreted for models with $\dot{E}_{\mathrm{dep}, 51}=120$ (black) and with $\dot{E}_{\mathrm{dep}, 51}=1.5$ (gray). (b) Ejected Fe mass (solid line: explosive nucleosynthesis products, dotted line: the jet contribution) as a function of the energy deposition rate. (c) Dependence of abundance ratio $[\mathrm{C} / \mathrm{Fe}]$ on the energy deposition rate.

$f_{\text {th }}$, thus being constant in time in the present models. On the other hand, $P_{\text {fall }}$ decreases with time, since the density of the outer materials decreases following the gravitational collapse (e.g. Fryer \& Mészáros 2003). For lower $\dot{E}_{\text {dep }}, P_{\text {jet }}$ is lower, so that the jet injection $\left(P_{\text {jet }}>P_{\text {fall }}\right)$ is realized at a later time when the central remnant becomes more massive due to more infall. As a result, the accreted region and $M_{\text {rem }}$ are larger for lower $\dot{E}_{\text {dep }}$.

A model with lower $\dot{E}_{\text {dep }}$ has larger $M_{\text {rem }}$, higher $[\mathrm{C} / \mathrm{Fe}]$, and smaller amount of Fe $[M(\mathrm{Fe})]$ because of the larger amount of fallback (Figs. 2bc, Tominaga et al. 2007a). The larger amount of fallback decreases the mass of the inner core relative to the mass of the outer layer. The fallback of the $\mathrm{O}$ layer also reduces $M(\mathrm{Fe})$ because $\mathrm{Fe}$ is mainly synthesized explosively in the $\mathrm{Si}$ and O layers. The variation of $\dot{E}_{\text {dep }}$ in the jet-induced explosions predicts that the variation of $[\mathrm{C} / \mathrm{Fe}]$ corresponds to that of $M(\mathrm{Fe})$.

\subsection{Comparison with the spherical supernova model}

The calculations of the jet-induced explosions show that the ejection of the inner matter is compatible with the fallback of the outer matter (Fig. 2a). This is consistent with the two-dimensional illustration of the mixing-fallback model (Fig. 3a) proposed by Umeda \& Nomoto (2002). The mixing-fallback model has three parameters; initial mass cut $\left[M_{\text {cut }}(\right.$ ini $\left.)\right]$, outer boundary of the mixing region $\left[M_{\operatorname{mix}}(\right.$ out $\left.)\right]$, and a fraction of matter ejected from the mixing region $(f)$. The remnant mass is written as

$$
M_{\text {rem }}=M_{\text {cut }}(\text { ini })+(1-f)\left[M_{\text {mix }}(\text { out })-M_{\text {cut }}(\text { ini) })\right] .
$$

The three parameters would relate to the hydrodynamical properties of the jet-induced explosion models, e.g. the inner boundary $\left(M_{0}\right)$, the outer edge of the accreted region $\left(M_{\text {acc,out }}\right)$, and the width between the edge of the accreted region and the jet axis.

We calculate a model with $\dot{E}_{\mathrm{dep}, 51}=120$ and $M_{0}=2.3 M_{\odot}\left(R_{0} \sim 3 \times 10^{3} \mathrm{~km}\right)$ to compare the yield of the jet-induced SN explosion with that of the spherical SN explosion. The inner boundary, the outer edge of the accreted region, and the central remnant mass of the jet-induced explosion model are $M_{0}=2.3 M_{\odot}, M_{\text {acc } \text {,out }}=12.2 M_{\odot}$, and $M_{\mathrm{rem}}=8.1 M_{\odot}$. Its abundance pattern is well reproduced by the spherical SN model with the same main-sequence mass $M_{\mathrm{ms}}=40 M_{\odot}$ and the explosion energy $E=3 \times 10^{52}$ ergs (Fig. 3b). The parameters of the mixing-fallback model are $M_{\text {cut }}\left(\right.$ ini) $=2.3 M_{\odot}$, 

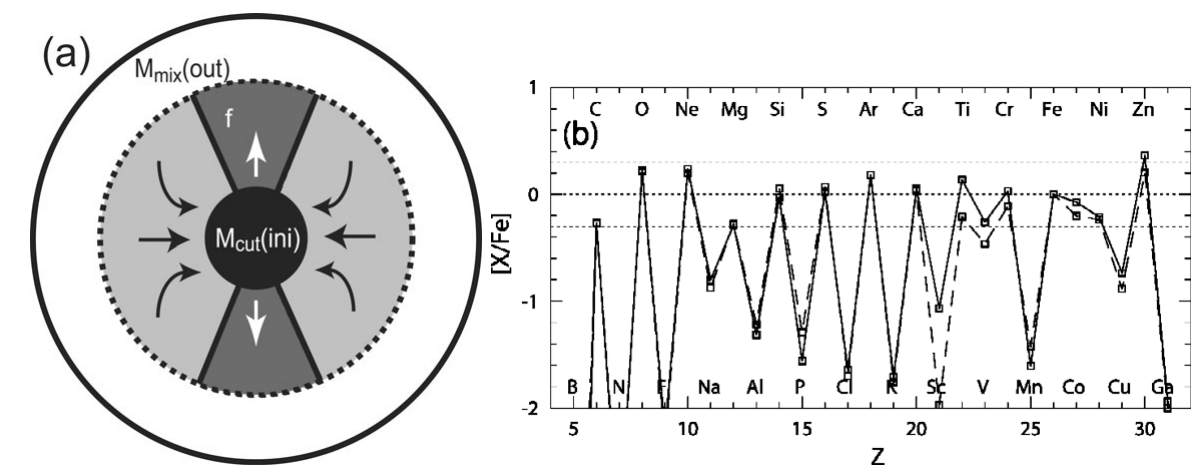

Figure 3. (a) Two-dimensional illustration of the mixing-fallback model. (b) Comparison of the abundance patterns of the jet-induced SN model with $\dot{E}_{\mathrm{dep}, 51}=120$ and $M_{0}=2.3 M_{\odot}($ solid line) and the mixing-fallback model (dashed line).

$M_{\text {mix }}($ out $)=10.8 M_{\odot}$ and $f=0.19$. The resultant $M_{\text {rem }}\left(=9.2 M_{\odot}\right)$ is slightly larger than $M_{\text {rem }}$ of the jet-induce SN model.

There, however, are some elements showing differences. The differences stem from the high-entropy explosion due to the concentration of the energy injection (e.g. Maeda \& Nomoto 2003). The enhancements of $[\mathrm{Sc} / \mathrm{Fe}]$ and $[\mathrm{Ti} / \mathrm{Fe}]$ improve agreements with the observations. Such a thermodynamical feature of the jet-induced explosion model cannot be reproduced by the mixing-fallback model. A "low-density" modification might mimic the high-entropy environment (e.g. Umeda \& Nomoto 2005; Tominaga et al. 2007b).

\section{Trends with Metallicity}

We calculate SN models with $M_{\mathrm{ms}}=13-50 M_{\odot}$. The explosion energies are set to be consistent with the observations of present SNe (e.g. Tanaka et al. 2008). The SN yields are compared with the abundance patterns of the EMP stars that show certain trends of abundance ratios $[\mathrm{X} / \mathrm{Fe}]$ with respect to $[\mathrm{Fe} / \mathrm{H}]$ (Cayrel et al. 2004).

The observed abundance ratios against $[\mathrm{Fe} / \mathrm{H}]$ are compared with yields of individual SN models and the Salpeter's IMF-integrated yield (Fig. 4). [Fe/H] of a next-generation star is determined by $M(\mathrm{Fe})$ and the swept-up $\mathrm{H}$ mass. Since the swept-up $\mathrm{H}$ mass is almost proportional to $E$ of the $\mathrm{SN},[\mathrm{Fe} / \mathrm{H}]$ of the next-generation stars are determined by a equation $[\mathrm{Fe} / \mathrm{H}]=\log _{10}\left[\frac{M(\mathrm{Fe})}{M_{\odot}} /\left(\frac{E}{10^{51} \mathrm{ergs}}\right)^{6 / 7}\right]-C$ (Thornton et al. 1998), where $C$ is assumed to be a constant value of 1.4. $[\mathrm{Fe} / \mathrm{H}]$ of the IMF-integrated abundance ratios are assumed to be same as normal SN models $([\mathrm{Fe} / \mathrm{H}] \sim-2.6)$.

HNe explode with $E \gtrsim 10^{52} \mathrm{ergs}$ and eject large amount of ${ }^{56} \mathrm{Ni}\left(\gtrsim 0.1 M_{\odot}\right)$, while normal SNe explode with $E \sim 10^{51} \mathrm{ergs}$ and eject $\sim 0.07 M_{\odot}$ of ${ }^{56} \mathrm{Ni}$. Therefore, according to the above equation, $[\mathrm{Fe} / \mathrm{H}]$ of a next-generation star originated from a $\mathrm{HN}$ is lower than that of a next-generation star originated from a normal SN. The higher-energy explosion raises explosive nucleosynthesis under higher entropy and thus leads higher [Zn/Fe]. This accompaniment explains the observed trends of $[\mathrm{Zn} / \mathrm{Fe}]$ against $[\mathrm{Fe} / \mathrm{H}]$. The trends of other elements are also reproduced by the variations of $M_{\mathrm{ms}}$ and $E$ (Fig. 4).

\section{Conclusion}

We focus on two interesting properties observed in the abundance patterns of the metalpoor stars: $(1)$ the higher $[\mathrm{C} / \mathrm{Fe}]$ for lower $[\mathrm{Fe} / \mathrm{H}]$ and $(2)$ the trends of $[\mathrm{X} / \mathrm{Fe}]$ against 


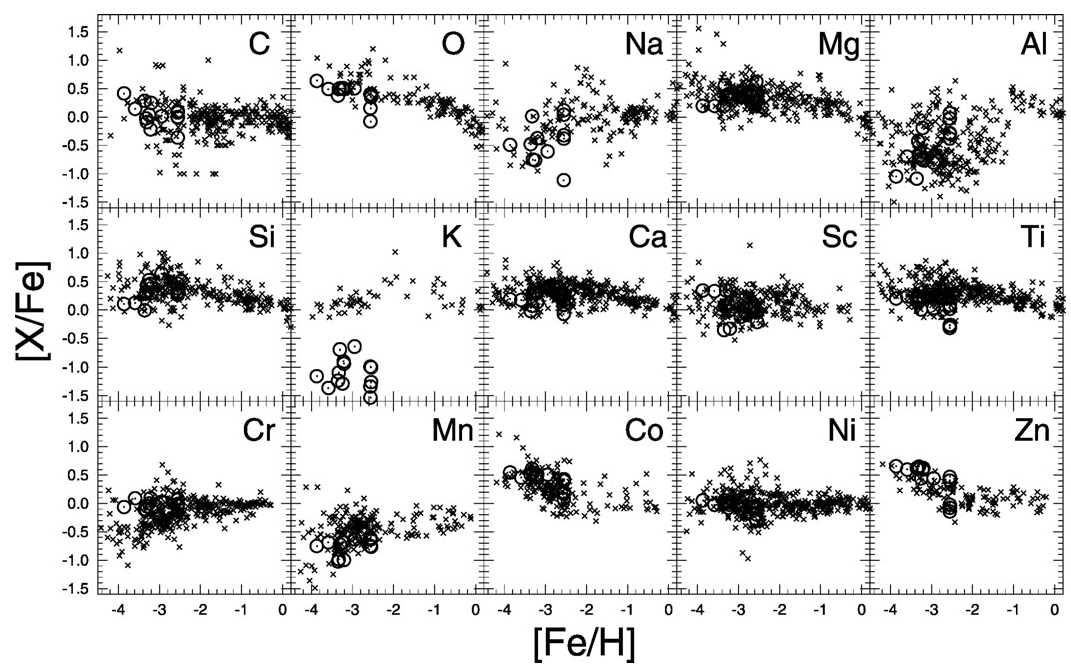

Figure 4. Comparison between the $[\mathrm{X} / \mathrm{Fe}]$ trends of observed stars (e.g. Cayrel et al. 2004; Honda et al. 2004: cross) and SN models with the mixing-fallback model and applied the $Y_{\mathrm{e}}$ and "low-density" modifications (Tominaga et al. 2007b: circles).

$[\mathrm{Fe} / \mathrm{H}]$. The variations of the metal-poor stars are explained by the variations of $\mathrm{SNe}$ that contribute the metal enrichment of the early universe. Especially, (1) the variation of the energy deposition rates explains the tendency of $[\mathrm{C} / \mathrm{Fe}]$ against $[\mathrm{Fe} / \mathrm{H}]$ and $(2)$ the variations of $M_{\mathrm{ms}}$ and $E$ explain the trends of $[\mathrm{X} / \mathrm{Fe}]$ against $[\mathrm{Fe} / \mathrm{H}]$. We propose that the abundance patterns of the metal-poor stars will provide additional constraints on the explosion mechanism of GRBs and SNe beyond the direct observations.

\section{References}

Amati, L., Della Valle, M., Frontera, F., et al. 2007, A\&A A, 463, 913

Beers, T.C. \& Christlieb, N. 2005, ARAA, 43, 531

Cayrel, R., Depagne, E., Spite, M., et al. 2004, A\&A, 416, 1117

Depagne, E., Hill, V., Spite, M., et al. 2002, A\&A, 390, 187

Frail, D.A., Kulkarni, S. R., Sari, R., et al. 2001, ApJ (Letters), 562, L55

Fryer, C. \& Mészáros, P. 2003, ApJ (Letters), 588, L25

Galama, T. J., Vreeswijk, P. M., van Paradijs, J., et al. 1998, Nature, 395, 670

Honda, S., Aoki, W., Kajino, T., et al. 2004, ApJ, 607, 474

Iwamoto, N., Umeda, H., Tominaga, N., Nomoto, K., \& Maeda, K. 2005, Science, 309, 451

Maeda, K., Nakamura, T., Nomoto, K., et al. 2002, ApJ, 565, 405

Maeda, K. \& Nomoto, K. 2003, ApJ, 598, 1163

Nagataki, S., Mizuta, A., \& Sato, K. 2006, ApJ, 647, 1255

Nomoto, K., Tominaga, N., Umeda, H., et al. 2006, Nucl. Phys. A, 777, 424 (astro-ph/0605725)

Tanaka, M., Tominaga, N., Nomoto, K., et al. 2008, ApJ, submitted (arXiv:0807.1674)

Thornton, K., Gaudlitz, M., Janka, H.-Th., \& Steinmetz, M. 1998, ApJ, 500, 95

Tominaga, N., Maeda, K., Umeda, H., et al. 2007a, ApJ (Letters), 657, L77

Tominaga, N., Umeda, H., \& Nomoto, K. 2007b, ApJ, 660, 516

Tominaga, N. 2007, ApJ, submitted (arXiv:0711.4815)

Umeda, H. \& Nomoto, K. 2002, ApJ, 565, 385

Umeda, H. \& Nomoto, K. 2005, ApJ, 619, 427

Woosley, S. E. \& Weaver, T. A. 1995, ApJS, 101, 181 